\title{
(6) OPEN ACCESS \\ Safety and efficacy of gravitational shunt valves in patients with idiopathic normal pressure hydrocephalus: a pragmatic, randomised, open label, multicentre trial (SVASONA)
}

\author{
Johannes Lemcke, ${ }^{1}$ Ullrich Meier, ${ }^{1}$ Cornelia Müller, ${ }^{2}$ Michael J Fritsch, ${ }^{2}$ Uwe Kehler, ${ }^{3}$ \\ Niels Langer, ${ }^{3}$ Michael Kiefer, ${ }^{4}$ Regina Eymann, ${ }^{4}$ Martin U Schuhmann, ${ }^{5}$ \\ Andreas Speil, ${ }^{5}$ Friedrich Weber, ${ }^{6}$ Victor Remenez, ${ }^{6}$ Veit Rohde, ${ }^{7}$ \\ Hans-Christoph Ludwig, ${ }^{7}$ Dirk Stengel ${ }^{8,9}$
}

For numbered affiliations see end of article.

\section{Correspondence to Dr J Lemcke and Prof} Dr U Meier, Department of Neurosurgery, Unfallkrankenhaus Berlin, Warener Str 7, Berlin 12683, Germany;

johannes.lemcke@ukb.de

Received 27 August 2012 Revised 8 January 2013 Accepted 5 February 2013 Published Online First

1 March 2013

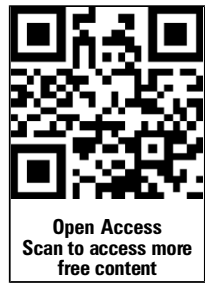

To cite: Lemcke J, Meier U, Müller C, et al. J Neurol Neurosurg Psychiatry 2013;84:850-858.

\section{ABSTRACT \\ Objectives To investigate whether gravitational valves reduce the risk of overdrainage complications compared with programmable valves in ventriculoperitoneal (VP) shunt surgery for idiopathic normal pressure hydrocephalus (iNPH). \\ Background Patients with iNPH may benefit from VP shunting but are prone to overdrainage complications during posture changes. Gravitational valves with tantalum balls are considered to reduce the risk of overdrainage but their clinical effectiveness is unclear. Methods We conducted a pragmatic, randomised, multicentre trial comparing gravitational with non- gravitational programmable valves in patients with iNPH eligible for VP shunting. The primary endpoint was any clinical or radiological sign (headache, nausea, vomiting, subdural effusion or slit ventricle) of overdrainage 6 months after randomisation. We also assessed disease specific instruments (Black and Kiefer Scale) and Physical and Mental Component Scores of the Short Form 12 (SF-12) generic health questionnaire.}

Results We enrolled 145 patients (mean (SD) age 71.9 (6.9) years), 137 of whom were available for endpoint analysis. After 6 months, 29 patients in the standard and five patients in the gravitational shunt group developed overdrainage (risk difference $-36 \%, 95 \% \mathrm{Cl}$ $-49 \%$ to $-23 \% ; p<0.001)$. This difference exceeded predetermined stopping rules and resulted in premature discontinuation of patient recruitment. Disease specific outcome scales did not differ between the groups although there was a significant advantage of the gravitational device in the SF-12 Mental Component Scores at the 6 and 12 month visits.

Conclusions Implanting a gravitational rather than another type of valve will avoid one additional overdrainage complication in about every third patient undergoing VP shunting for iNPH.

\section{INTRODUCTION}

Idiopathic normal pressure hydrocephalus (iNPH) is the only variant of dementia disorders possibly treatable by neurosurgical intervention. iNPH is a neurodegenerative condition clinically characterised by gait ataxia, urinary incontinence and memory disturbance (the so-called Hakim's triad). ${ }^{1}$
In contrast with other types of dementia, ataxia represents an early and possibly pathognomic sign of iNPH. Neuroimaging typically shows dilated ventricles in the absence of increased intracranial pressure. Current pathophysiological models attribute iNPH to a complex dysfunction of cerebral blood flow with accompanying changes in CSF physiology rather than a simple imbalance of liquor production and resorption. ${ }^{2}$

The precise epidemiology of iNPH in industrialised countries remains to be defined. Data from Norway suggest an overall 5 year incidence of 1.1/ 100000 , which may increase to $30.2 / 100000$ in subjects over 65 years of age. ${ }^{3}$

Ventriculoperitoneal (VP) shunt surgery is a widely established intervention for iNPH although there is no conclusive evidence from controlled trials demonstrating superior outcomes of VP shunting over possible alternative treatment options (eg, endoscopic third ventriculostomy (ETV), or even watchful waiting). ${ }^{4}$ In the Hydrocephalus Association Survey 2003-2005, patients who underwent surgery had a non-significant reduction in the relative risk (RR) of dependent living compared with non-operative management $(14 / 185$ vs 6/66, RR $0.83,95 \%$ CI 0.33 to 2.08 ). While more shunt patients reported improved health related quality of life, they required heightened care compared with endoscopic third ventriculostomy patients. ${ }^{6}$

VP shunting carries the risk of overdrainage complications, such as hygroma and subdural bleeding, which may cause severe headaches and nausea, subsequently demanding revision surgery.

The only current randomised trial on this condition, the Dutch Hydrocephalus Study, showed that low pressure valves lead to better neurological outcomes than medium pressure valves but are associated with a 3.3 (95\% CI 1.6 to 6.9) times higher RR of chronic subdural effusions. ${ }^{7}$

The key problem of all drainage concepts is the posture dependent hydrostatic pressure change in a VP shunt. If the valve is programmed to provide adequate intraventricular and shunt pressure with the patient in the supine position, it may rapidly change to overdrainage in the upright position. If 
the valve pressure is set too low with the patient standing, underdrainage may occur in the horizontal position, compromising the beneficial effect of shunt surgery.

Recently, gravitational ball-in-cone units have been developed to overcome this siphoning effect by switching between a low pressure mode in the supine position and a high pressure mode in the upright position. ${ }^{8-10}$

Although plausible, it is unclear whether the more expensive gravitational valves improve the risk-benefit ratio of shunt surgery in iNPH patients.

We hypothesised that gravitational devices reduce the risk of overdrainage complications while, at the same time, they maintain the efficacy of conventional valves. To prove this hypothesis, we conducted a multicentre randomised trial of VP shunting with either a gravitational or a non-gravitational valve in patients with iNPH.

\section{METHODS}

\section{General remarks}

The SVASONA (Shunt Valves plus shunt Assistant versus Shunt valves alone for controlling Overdrainage in idiopathic Normal pressure hydrocephalus in Adults) study was a pragmatic, multicentre, open label, randomised, parallel group trial conducted at seven centres in Germany. The trial investigated two different concepts of surgical CSF drainage in iNPH-that is, posture adapted CSF drainage using a gravitational unit versus shunting by a programmable valve. The primary objective of the study was to assess whether gravitational valve shunts can significantly reduce overdrainage complications compared with programmable valves, thereby improving the therapeutic index of shunt surgery for iNPH. Secondary objectives were to evaluate neurological recovery and the health related quality of life of patients undergoing either procedure.

In the planning phase of the trial, we searched PubMed Medline, Embase and the Cochrane Library with the terms 'normal pressure hydrocephalus', 'shunt"', 'surg*', 'valve*' and 'outcome" for randomised trials as well as systematic reviews, for any available evidence to answer our research question. We also reviewed the databases of clinicaltrials.gov and Current Controlled Trials (ISRCTN) for ongoing studies on the topic.

We could not identify any other published or currently recruiting trial comparing standard programmable and gravitational valves in a head to head fashion for the surgical treatment of the disease of interest. This lack of evidence from randomised controlled trials supported the need for this trial, and stressed that the equipoise principle was met.

\section{Participants}

We enrolled consecutive patients who gradually developed gait disturbance unexplainable by other health conditions, and showed at least one other clinical sign of the Hakim's triad suggesting the presence of iNPH (dementia and incontinence). ${ }^{11}$

Eligible patients had to demonstrate a communicating hydrocephalus with enlarged lateral ventricles, equalling an Evans Index of 0.3 or higher, as verified by CT or MRI. ${ }^{12}$ The Evans Index is defined as the maximum frontal horn ventricular width divided by the transverse inner diameter of the skull in the same slice. A ratio of 0.3 or higher represents the classic threshold for diagnosing ventriculomegaly.

Also, at least one positive result out of the following pattern of invasive tests was required for patient inclusion: (1) resistance to outflow $\left(\mathrm{R}_{\text {out }}\right)$ of $13 \mathrm{~mm} \mathrm{Hg} / \mathrm{min} \times \mathrm{ml}$ or more in the lumbar CSF infusion test, ${ }^{13-18}$ (2) clinical improvement after a lumbar spinal tap or (3) positive B wave analysis. ${ }^{17}$ 19-22
We excluded patients with secondary NPH due to bleeding or infection, cerebral parenchymal lesions on CT or MRI scans, or any contraindication for surgery. Patients were also excluded if, according to the screening physician's judgment, they were unlikely to attend the planned follow-up visits. This included long distance travel, impaired mobility, low compliance or a presumed limited remaining lifespan.

The trial protocol was approved by the institutional review boards of the coordinating centre (Charité Medical University Centre, Berlin, Germany, EA1/165/06) and all collaborating institutions. The study was conducted in accordance with Good Clinical Practice and federal medical device regulations. All devices employed in the study were approved for clinical use under European laws.

\section{Randomisation}

After obtaining written informed consent from patients or their closest relatives, participants were randomised in a 1:1 fashion to either treatment group using a block randomisation plan (using the http://www.randomization.com online random sequence generator). Computer generated random lists and sealed envelopes were prepared by the central statistical unit and distributed to the collaborating institutions. For practical reasons and because of varying access to high speed internet connections in the operating theatres of the collaborating centres, we preferred the envelope approach over a web based randomisation tool. Sealed envelopes were opened immediately before surgery in the operating theatre. A treatment allocation sheet signed by a surgeon was faxed to the coordinating site to ensure that treatment assignment at individual centres matched the original randomisation sequence.

\section{Procedures}

All patients underwent implantation of a silicone VP shunt under general anaesthesia carried out by experienced surgeons accredited for the study, using programmable units with similar hydrodynamic characteristics. ${ }^{23} 24$ Participants were randomly assigned to receive either a gravitational device (proGAV, Aesculap-Miethke, Potsdam, Germany) or a programmable valve (CMPV, Codman and Shurtleff, Johnson and Johnson, Ryanham, Massachusetts, USA).

Valves were implanted with an opening pressure of $100 \mathrm{~mm}$ $\mathrm{H}_{2} \mathrm{O}$. Three months after surgery they were adjusted to the low pressure range $\left(70 \mathrm{~mm} \mathrm{H}_{2} \mathrm{O}\right)$. This was done because the Dutch iNPH study suggested better outcomes in patients with low pressure valves compared with medium high pressure valves. The decision about opening pressure thresholds of gravitational units was taken at the discretion of the collaborating centres. Manufacturers' recommendations were adhered to throughout the process.

Trial specific visits and data collection were scheduled at baseline, discharge, and after 6 and 12 months of follow-up. Available resources for this trial prohibited a tighter follow-up scheme, as well as an expansion of the observation period. All patients underwent a baseline cranial CT as well as the required CT scan 6 months after randomisation. Additional CT or MRI scans were ordered at the discretion of local investigators.

Changes in neurological status were assessed with two disease specific outcome tools ${ }^{25}$ : the Black Grading Scale and the Kiefer Score.

The 6 point Black Scale introduced in 1980 classifies outcomes as excellent (resumed pre-illness activity without deficit), good (resumed pre-illness activity with moderate deficit), fair (improved, but no return to previous work), transient 
(temporary major improvement), poor (no change or worse) and dead (deceased within 6 weeks after surgery or as a result of surgery).

The Kiefer Score attempts to grade the severity of the three key symptoms of iNPH (mental deficits, gait disturbance, incontinence) and two additional minor symptoms (headache and dizziness). ${ }^{26}$ The overall score may reach values between 0 and 24, with higher scores indicating more severe impairment. ${ }^{27}$

We also evaluated health related quality of life with the generic Short Form 12 V2.0 (SF-12) questionnaire, calculating population norm based Physical (PCS) and Mental (MCS) Component Scores.

The central coordinating unit prepared electronic case report forms (CRF) run on study specific laptop computers at the different trial sites. Two research assistants were responsible for telephone and on site monitoring of data during the study and after database closure.

The primary trial endpoint was any overdrainage complication occurring within 6 months after randomisation, as determined by local investigators. The diagnosis of overdrainage was verified by two neurosurgeons at the coordinating centre based on a consensus review of CRF entries and CT reports. Overdrainage was defined as any clinical symptom suggestive of overdrainage (headache, nausea, vomiting) requiring readjustment of the valve to a pressure of $90 \mathrm{~mm} \mathrm{H}_{2} \mathrm{O}$ or more, the presence of subdural hygroma or haematoma with a thickness of at least $3 \mathrm{~mm}$, a slit ventricle in CT scans or subsequent crossover from the programmable to the gravitational device.

Categorical secondary endpoints were surgical revision resulting from any cause, surgical site infections, ventriculitis and underdrainage. The latter was defined as ongoing ventricle enlargement accompanied by a secondary increase in symptoms.

Ordinal and continuous secondary endpoints included raw values and longitudinal changes in Black Scales, Kiefer Scores, SF-12 PCS and MCS, and the Evans Index.

The SVASONA trial compared approved and established devices. Adverse events and serious adverse events were recorded cumulatively and evaluated for their association with the procedure and devices under investigation by a panel of investigators at regular study meetings.

\section{Statistical analysis}

All endpoints were analysed on an intention to treat basis. Only intention to treat results are presented here.

In the Dutch Normal Pressure Hydrocephalus Study, the incidence of all subdural effusions was 51/96, with 31 effusions being permanent $(32 \%, 95 \%$ CI $23 \%$ to $46 \%){ }^{7}$ A later case series showed a similar overall incidence of symptomatic overdrainage complications with the standard of care, the programmable Codman-Hakim valve, of 189/583 (32\%, 95\% CI 29\% to $36 \%){ }^{28}$ For this study, we assumed a control event rate of $25 \%$. A risk reduction of $15 \%$ was considered realistic and clinically relevant by the panel of investigators. To demonstrate this difference with a power of $80 \%$ (plus a power reserve of $3 \%$ ) and a two sided $\alpha$ error of 5\%, 123 patients had to be evaluated in either group. As an early stopping rule, the trial would have had to be discontinued prematurely if the upper or lower $\mathrm{z}$ values for the difference in proportion exceeded 2.96 or -2.96 , or the $\mathrm{p}$ value was lower than 0.0031 at the time of the interim analysis after inclusion of $50 \%$ of the target sample.

Results are presented as numbers, proportions, means and medians, according to the underlying distribution of the data. Differences between groups are expressed as risk differences, risk ratios (RR) and mean differences. Estimates of precision include ranges, interquartile ranges, SDs and 95\% CI.

For confirmatory analysis of the primary endpoint, we used a generalised linear model to assess differences in proportions with a binomial variance and the logit link function. This model was also used to determine differences in secondary binary endpoints and to adjust the estimates for centre effects and key demographic variables (age, sex, body mass index (BMI), the American Society of Anaesthesiologists physical status classification system (ASA) and the Charlson Comorbidity Index).

Longitudinal changes in radiological measures, and disease specific and generic quality of life assessment instruments were evaluated by analysis of variance. The STATA 11 statistical software package (StataCorp LP, College Station, Texas, USA) was used for all analyses.

\section{RESULTS}

A total of 145 patients were enrolled in the study. Complete data were available for 137 participants at the 6 month follow-up date. The observed risk difference in overdrainage events exceeded the expected effect size $(z=-4.1)$ and the statistical discontinuation limit. Consequently, the investigators decided to terminate the trial at this stage. One slow recruiting trial centre did not comply with the protocol and so five patients originally enrolled at this centre were excluded from further analysis (figure 1).

Eligible participants in the study (89 men and 56 women) had a mean age of 71.9 years (SD 6.9; range 44 to 83 years). Baseline demography was similar in the groups, except for a slightly higher number of men randomly assigned to the nongravitational device group (table 1 ).

At the 6 month follow-up visit, 26 patients in the programmable valve group and four patients in the gravitational shunt group showed signs and symptoms of overdrainage (risk difference $-33 \%, 95 \%$ CI $-46 \%$ to $-20 \%$; $<<0.001)$. Up until then, 16 patients $(23 \%, 95 \%$ CI $13 \%$ to $34 \%)$ had already been or were scheduled to be implanted with a gravitational device. The cumulative incidence of overdrainage events, recorded from the index procedure to the trial visit 6 months after randomisation, was 29 and five, respectively (risk difference $-36 \%,-49$ to $-23 \%$; $\mathrm{p}<0.001)$. Trial centre allocation, male sex, age, ASA class, Evans Index, Charlson comorbidity severity, cortical atrophy grade, intracranial pressure or duration of surgery did not influence outcomes by themselves, as evaluated by general linear models.

The advantage of the gravitational valve over the programmable valve in reducing overdrainage held up until the 12 month follow up (table 2). Two patients in the experimental trial arm died for reasons unrelated to the intervention.

Overall, the incidence of adverse events was low. No supposed unexpected serious adverse reactions, in particular no device related adverse events, were observed during the trial period.

The morphological indicator of ventricle enlargement, the Evans Index, decreased over time $(p=0.001)$ but was unrelated to the treatment group $(p=0.537)$ (figure 2$)$. The disease specific Black Scale did not change between the 6 and 12 month assessments (mean difference $0.15,95 \% \mathrm{CI}-0.06$ to 0.36 ). In contrast, the Kiefer Scale decreased after shunting $(p<0.001)$ without showing a significant difference between treatment groups $(\mathrm{p}=0.339)$ (figure 3$)$.

SF-12 PCS improved over time, again without a difference between implant type. It is of note that MCS showed better ratings $(p<0.001)$ in the gravitational group after 6 months (figure 4). 
Figure 1 Trial profile and patient selection procedure according to CONSORT recommendations. ITT, intention to treat.

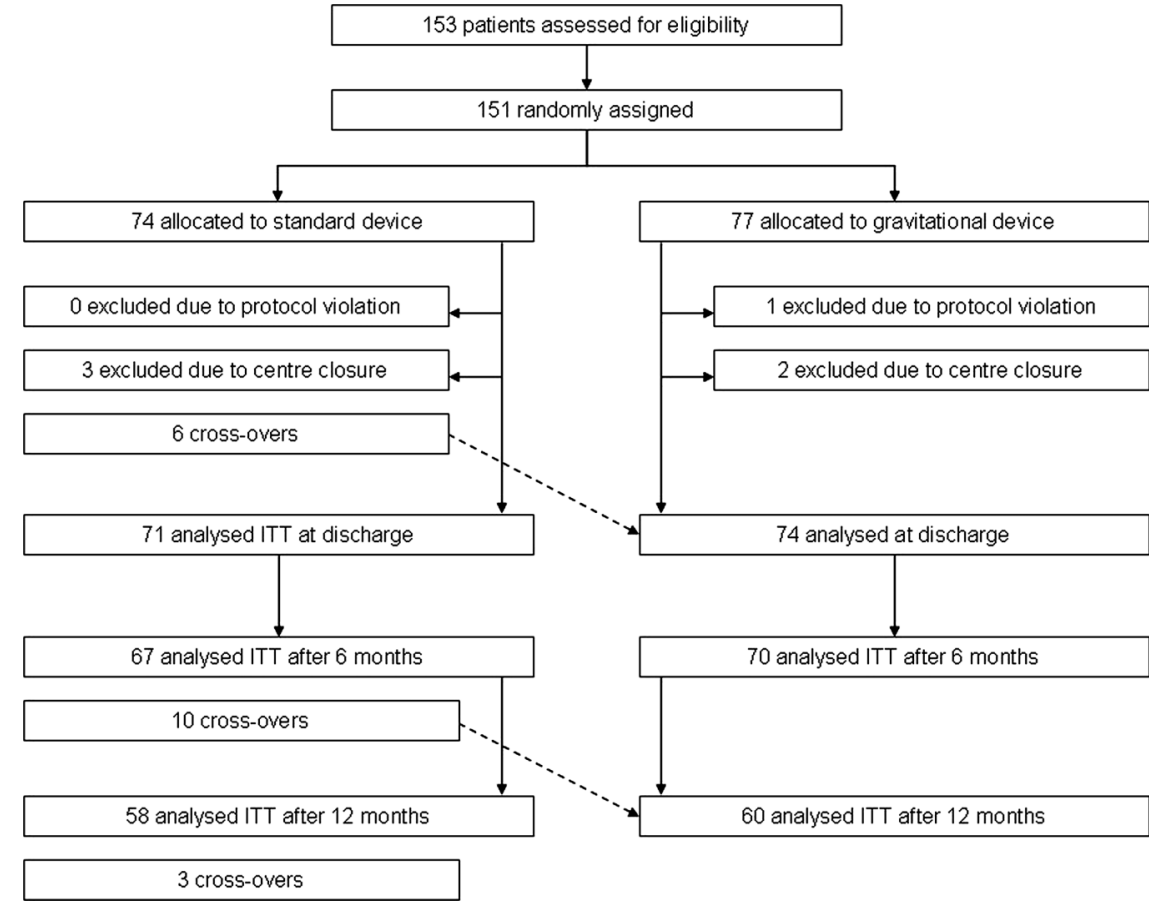

\section{DISCUSSION}

The key finding of this multicentre trial is that, if shunt surgery is considered the treatment option of choice for patients with confirmed iNPH, it should be performed using a gravitational valve. The latter may prevent one additional overdrainage complication in every third patient undergoing shunting with a gravitational compared with a non-gravitational programmable valve.

Table 1 Baseline characteristics of the patients included in the SVASONA trial

\begin{tabular}{|c|c|c|}
\hline & Programmable valve group $(n=71)$ & Gravitational valve group $(n=74)$ \\
\hline \multicolumn{3}{|l|}{ Sex } \\
\hline Male (n (\%)) & $48(68)$ & $41(55)$ \\
\hline Female (n (\%)) & $23(32)$ & $33(45)$ \\
\hline Age at surgery (years) (mean (SD)) & $71.2(7.0)$ & $72.7(6.7)$ \\
\hline BMI (mean (SD)) & $28.6(4.2)$ & $27.1(4.1)$ \\
\hline Evans Index (mean (SD)) & $0.38(0.06)$ & $0.37(0.05)$ \\
\hline Cortical atrophy, any grade $(\mathrm{n}(\%))$ & $61(86)$ & $67(91)$ \\
\hline Mean intracranial pressure $\left(\mathrm{mm} \mathrm{H}_{2} \mathrm{O}\right)$ (mean (SD)) & $9.6(3.6)$ & $9.6(3.1)$ \\
\hline ASA III patients (n (\%)) & $23(32)$ & $25(34)$ \\
\hline \multicolumn{3}{|l|}{ Charlson Comorbidity Index (n) } \\
\hline Not indicated & 8 & 7 \\
\hline 0 & 18 & 24 \\
\hline 1 & 20 & 20 \\
\hline 2 & 9 & 11 \\
\hline 3 & 7 & 7 \\
\hline 4 & 7 & 5 \\
\hline$>4$ & 2 & 0 \\
\hline Duration of surgery (min) (mean (SD)) & $60.5(22.5)$ & $61.9(23.7)$ \\
\hline Duration of hospital stay (days) (mean (SD)) & $7.9(4.3)$ & $7.1(3.9)$ \\
\hline \multicolumn{3}{|l|}{ Gravitational unit opening pressure $\left(\mathrm{mm} \mathrm{H}_{2} \mathrm{O}\right)(\mathrm{n})$} \\
\hline 200 & & 20 \\
\hline 250 & & 34 \\
\hline 300 & & 16 \\
\hline 350 & & 4 \\
\hline Postoperative CT scan (n (\%)) & $70(99)$ & $71(96)$ \\
\hline Interval to postoperative CT (days) (mean (SD)) & $3.9(3.7)$ & $4.2(4.5)$ \\
\hline
\end{tabular}


Table 2 Outcome by endpoint

\begin{tabular}{|c|c|c|c|c|c|}
\hline \multirow[b]{2}{*}{ Assessment } & \multicolumn{2}{|l|}{ Valve } & \multirow[b]{2}{*}{ Risk difference $(95 \% \mathrm{Cl})$} & \multirow[b]{2}{*}{ OR $(95 \% \mathrm{Cl})^{*}$} & \multirow[b]{2}{*}{ p Value } \\
\hline & Programmable & Gravitational & & & \\
\hline \multicolumn{6}{|l|}{ Hospital stay (t1) } \\
\hline Participants & 71 & 74 & & & \\
\hline Subdural effusion & 10 & 1 & $-13 \%(-21 \%$ to $-4 \%)$ & 0.08 (0.01 to 0.67$)$ & 0.020 \\
\hline Bleeding complications & 4 & 1 & $-4 \%(-10 \%$ to $2 \%)$ & $0.23(0.03$ to 2.10$)$ & 0.193 \\
\hline Misplacement & 6 & 4 & $-3 \%(-11 \%$ to $5 \%)$ & 0.62 (0.17 to 2.29 ) & 0.473 \\
\hline \multicolumn{6}{|l|}{ Follow-up at 6 months (t2) } \\
\hline Participants & 67 & 70 & & & \\
\hline Subdural effusion & 24 & 4 & $-30 \%(-43 \%$ to $-17 \%)$ & $0.11(0.04$ to 0.33$)$ & $<0.001$ \\
\hline Overdrainage & 26 & 4 & $-33 \%(-46 \%$ to $-20 \%)$ & $0.10(0.03$ to 0.29$)$ & $<0.001$ \\
\hline Cumulative incidence of overdrainage & 29 & 5 & $-36 \%(-49 \%$ to $-23 \%)$ & $0.10(0.04$ to 0.28$)$ & $<0.001$ \\
\hline Underdrainage & 3 & 2 & $-2 \%(-8 \%$ to $5 \%)$ & 0.62 (0.10 to 3.82$)$ & 0.604 \\
\hline \multicolumn{6}{|l|}{ Follow-up at 12 months (t3) } \\
\hline Participants & 58 & 60 & & & \\
\hline Subdural effusion & 12 & 0 & $-21 \%(-31 \%$ to $-10 \%)$ & - & \\
\hline Overdrainage & 14 & 2 & $-21 \%(-33 \%$ to $-9 \%)$ & $0.10(0.02$ to 0.46$)$ & 0.003 \\
\hline Cumulative incidence of overdrainage & 34 & 7 & $-44 \%(-59 \%$ to $-30 \%)$ & $0.10(0.04$ to 0.24$)$ & $<0.001$ \\
\hline Underdrainage & 8 & 1 & $-12 \%(-21 \%$ to $-3 \%)$ & $0.00(0.01$ to 0.84$)$ & 0.033 \\
\hline \multicolumn{6}{|l|}{ Cumulative incidence of adverse events } \\
\hline Wound infection & 1 & 2 & $1 \%(-3 \%$ to $6 \%)$ & 1.94 (0.17 to 21.93$)$ & 0.591 \\
\hline Local bleeding & 4 & 1 & $-4 \%(-10 \%$ to $2 \%)$ & $0.23(0.03$ to 2.10$)$ & 0.193 \\
\hline Malplacement & 6 & 4 & $-3 \%(-11 \%$ to $5 \%)$ & $0.62(0.17$ to 2.29$)$ & 0.473 \\
\hline Device exchange & 3 & 1 & $-3 \%(-8 \%$ to $2 \%)$ & $0.31(0.03$ to 3.06$)$ & 0.316 \\
\hline Pressure adjustment & 29 & 28 & $-3 \%(-19 \%$ to $13 \%)$ & $0.88(0.45$ to 1.72$)$ & 0.711 \\
\hline Death & 0 & 2 & $3 \%(-1 \%$ to $6 \%)$ & - & \\
\hline
\end{tabular}

${ }^{*}$ Derived from generalised linear model, unadjusted data.

This assumption is mainly supported by surrogate measures (ie, overdrainage complications) although our results also suggest an advantage of gravitational valves over other programmable valves in the mental domains of health related quality of life.

While the observed point estimate of overdrainage incidence in the control group increased compared with previous reports by about $10 \%{ }^{7} 95 \%$ CI of proportions still overlapped. Thus there is currently no divergence of the present from the available body of evidence.
The present data may not help neurosurgeons in counselling patients and their relatives to opt for or against a surgical intervention. However, they may guide healthcare professionals in choosing the most appropriate device to avoid secondary interventions. The additional costs of gravitational units are likely to be offset by savings in later care, although this assumption needs to be confirmed by health economy studies, formally investigating the incremental cost effectiveness ratio and the dominant treatment strategy.

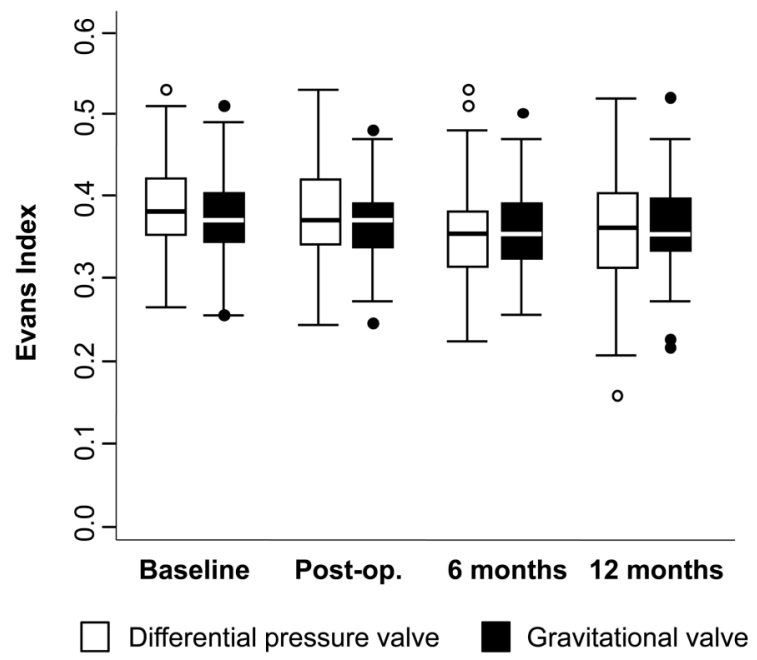

Figure 2 Longitudinal changes in ventricular enlargement, as indicated by the Evans Index.

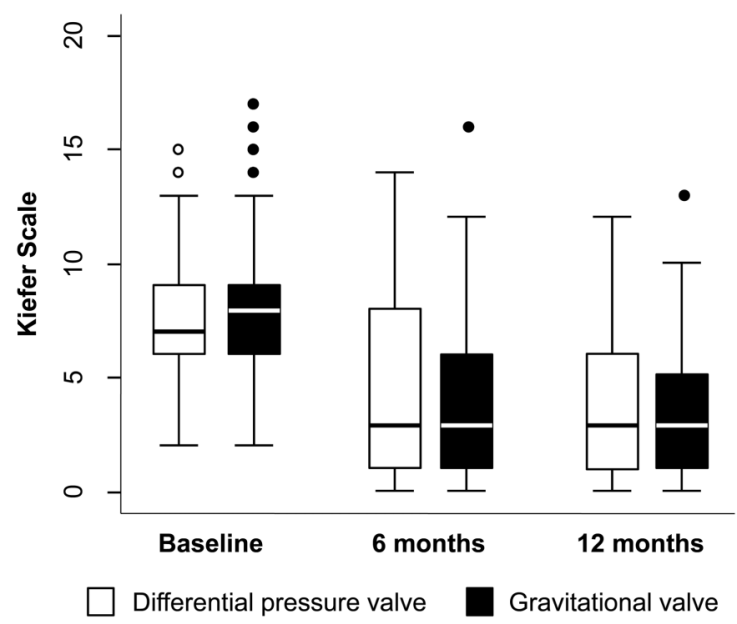

Figure 3 Disease specific outcomes by time and treatment group measured by the Kiefer Scale. 
Figure 4 Generic health outcomes as assessed by the Short Form 12 (SF-12) and its physical (PCS) and mental (MCS) Component Scores.

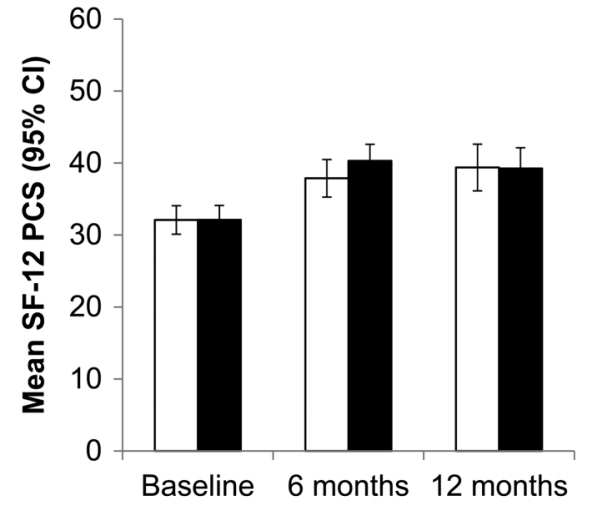

口Differential pressure valve - Gravitational valve

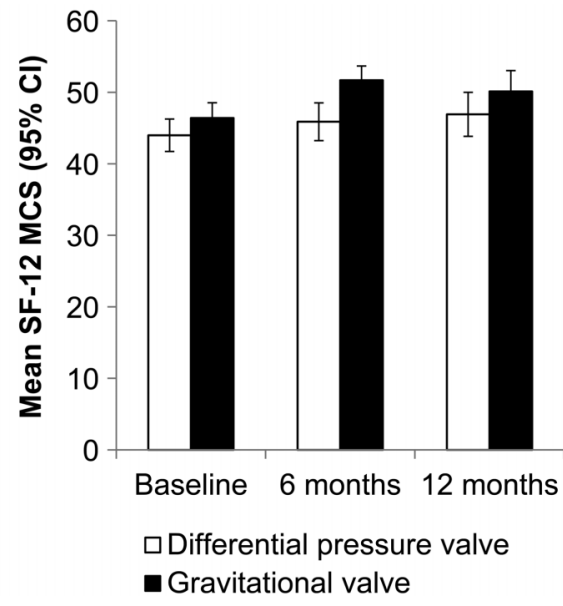

Stroop Test and Auditory Verbal Learning Test to monitor treatment effects after shunting. ${ }^{33}$

Assessment of the three key domains of health related quality of life (ie, physical, mental, social) in patients with dementia is challenging, and common generic instruments such as the Short Form family (ie, SF-36, SF-12, SF-8) or the Euro-Quality of Life 5D (EQ-5D) may fail in this setting. ${ }^{34}$ Differences in the reliability (or internal consistency, the ability of an instrument to measure something the same way twice) between the eight domains of the SF36 have been observed among elderly patients with (Mini-Mental State Examination score $\leq 23$ ) and without (Mini-Mental State Examination score $>24$ ) cognitive impairment. ${ }^{35}$ The advantage of the SF-12 used in the SVASONA trial is its simplicity, and the availability of normalised PCS and MCS. The values allow for a basic comparison of the health status of the study sample to a gender and age matched norm population. Altogether, the observed trends in Kiefer, Black and SF-12 PCS give confidence that neurological outcomes between the valve types under investigation are comparable.

The observation of normalisation of SF-12 MCS in the gravitational valve group, and consistently higher ratings compared with the programmable valve 6 and 12 months after randomisation, were unexpected. Effect sizes d (ie, mean differences divided by the pooled SD) were moderate $(d=0.62$ and $d=0.31$, respectively). These findings must, however, be interpreted with caution, given that the SF-12 is currently not validated in patients with iNPH and other types of dementia.

Further limitations of this study merit discussion. First, despite its multicentre design, the results may not be applicable to centres outside Europe. Regression analysis did not reveal differences between participating units with regard to the primary trial endpoint. However, we cannot exclude residual selection bias and centre effects because of the small sample size and premature discontinuation of patient enrolment. Second, we had to withdraw one centre from the study because of protocol violations, as revealed by rigorous monitoring. Although this deviation only affected five patients, it may have also resulted in selection bias. Third, a higher number of male patients were randomly assigned to the experimental group. Exploratory regression analysis did not indicate a significant impact of gender on primary trial outcomes. However, this imbalance may point to residual bias not eliminated by randomisation. Fourth, the available resources precluded more frequent and longer longitudinal assessments (eg, after 3 months and beyond 1 year). Thus we cannot make any statements about the subsequent risk of underdrainage in the gravitational trial arm after database 
closure. However, our data collected up to 1 year after randomisation did not suggest a higher, but rather a lower, rate of underdrainage with gravitational valves. Further studies are needed to investigate whether potential short term and mid term benefits of gravitational valves are offset by a higher risk of underdrainage in the long term.

A more technical issue of concern may be the chosen valve setting. The Dutch NPH study, our intellectual starting point prompting the SVASONA trial, clearly demonstrated that low pressure valves (set at $40 \mathrm{~mm} \mathrm{H}_{2} \mathrm{O}$ ) lead to significantly better outcomes than medium high pressure valves. Consequently, we aimed for a trial design with a general low pressure setting. On the other hand, it was obvious that the implantation of $50 \mathrm{~mm}$ $\mathrm{H}_{2} \mathrm{O}$ valves with and without a gravitational unit would cause hazardous overdrainage situations in many patients in the treatment arm without gravitational units. Thus we allowed for a slow adaptation from a high to a low pressure range, initially implanting both valves with $100 \mathrm{~mm} \mathrm{H}_{2} \mathrm{O}$ and later adjusting them to $70 \mathrm{~mm} \mathrm{H} \mathrm{H}_{2} \mathrm{O}$ after 3 months. We admit that this scheme is a compromise but it is still in accordance with manufacturers' recommendations and clinical practice.

Choosing the appropriate opening pressure of the gravitational unit remains a critical treatment step which was set according to the patient's body mass index. The rationale behind this is that the level of compensation for hydrostatic pressure changes ultimately depends on the height of the upper body and peritoneal pressure. However, only the hydrostatic difference between the upright and recumbent position can accurately be calculated. One may speculate whether overdrainage rates with gravitational units can further be lowered if it were possible to exactly determine the required degree of hydrostatic compensation.

In summary, gravitational valves showed a significant reduction in the incidence of surrogate markers of overdrainage up to 1 year following shunt surgery. While maintaining therapeutic efficacy and effectiveness (measured by disease specific outcome instruments such as the Black and Kiefer Scale), gravitational valves may have a superior therapeutic index compared with non-gravitational programmable valves. Possible advantages for mental components of health related quality of life demand further investigation. Health economy studies are ultimately needed to define the dominant treatment strategy, and to determine the most cost effective standard of care in patients with iNPH.

\section{Author affiliations \\ ${ }^{1}$ Department of Neurosurgery, Unfallkrankenhaus Berlin, Berlin, Germany ${ }^{2}$ Department of Neurosurgery, Ernst-Moritz-Arndt-University of Greifswald, Greifswald, Germany \\ ${ }^{3}$ Department of Neurosurgery, Asklepios Hospital Altona, Hamburg, Germany ${ }^{4}$ Department of Neurosurgery, Saarland Medical University, Homburg/Saar, Germany ${ }^{5}$ Department of Neurosurgery, Eberhard-Karls-University of Tübingen, Tübingen, Germany \\ ${ }^{6}$ Department of Neurosurgery, Hospital Cologne-Merheim, Cologne, Germany ${ }^{7}$ Department of Neurosurgery, Georg-August-University of Göttingen, Göttingen, Germany \\ ${ }^{8}$ Department of Trauma and Orthopaedic Surgery, Centre for Clinical Research, Unfallkrankenhaus Berlin, Berlin, Germany \\ ${ }^{9}$ Julius Wolff Institute, Charité Medical University Centre, Germany}

Acknowledgements We thank Annette Luckmann, MA, for editorial assistance. We also thank Kathleen Füssler, MSC, and Antje Spranger, SN, for assistance in data collection and monitoring.

Contributors JL, UM and DS designed the study. JL, UM, CM, MJF, UK, NL, MK, RE, MUS, AS, FW, VRe, VRo and H-CL collected the data. JL and DS were involved in quality control of the database and monitoring. DS conducted the statistical analyses. DS and JL drafted the manuscript. UM is the guarantor of this study. All authors contributed to the interpretation of the data and read and approved the final version of the report.
Funding This was an investigator initiated trial sponsored by Aesculap AG, Tuttlingen, Germany. The sponsors had no role in the study design, data collection, data analysis, data interpretation or writing of the report. The corresponding authors had complete access to all of the data obtained in the study and had final responsibility for the decision to submit the article for publication.

Competing interests DS is a board member of the German Trauma Association and received research grants as well as payments for lectures, travel and expert testimonies from the Federal Statutory Accident Insurance (DGUV), Biomet, DePuy, Stryker, AO Foundation, Federal Ministry of Education and Research (BMBF), and the European Commission outside the submitted work.

Clinical Trial Registration Information ISRCTN Number ISRCTN51046698.

Ethics approval The trial protocol was approved by the institutional review boards of the coordinating centre (Charité Medical University Centre, Berlin, Germany, EA1/165/06) and all collaborating institutions.

Provenance and peer review Not commissioned; externally peer reviewed.

Data sharing statement The SVASONA investigators are willing to share data for systematic reviews and meta-analyses, HTA reports and clinical practice guidelines.

Open Access This is an Open Access article distributed in accordance with the Creative Commons Attribution Non Commercial (CC BY-NC 3.0) license, which permits others to distribute, remix, adapt, build upon this work non-commercially, and license their derivative works on different terms, provided the original work is properly cited and the use is non-commercial. See: http://creativecommons.org/ licenses/by-nc/3.0/

\section{REFERENCES}

1 Hakim CA, Hakim R, Hakim S. Normal-pressure hydrocephalus. Neurosurg Clin N Am 2001;12:761-73.

2 Greitz D. Radiological assessment of hydrocephalus: new theories and implications for therapy. Neurosurg Rev 2004;27:145-65.

3 Brean A, Fredo HL, Sollid S, et al. Five-year incidence of surgery for idiopathic normal pressure hydrocephalus in Norway. Acta Neurol Scand 2009;120:314-16.

4 Gangemi M, Maiuri F, Buonamassa S, et al. Endoscopic third ventriculostomy in idiopathic normal pressure hydrocephalus. Neurosurgery 2004;55:129-34.

5 Bergsneider $M$, Black PM, Klinge $P$, et al. Surgical management of idiopathic normal-pressure hydrocephalus. Neurosurgery 2005;57:S29-39.

6 Cage TA, Auguste KI, Wrensch M, et al. Self-reported functional outcome after surgical intervention in patients with idiopathic normal pressure hydrocephalus. J Clin Neurosci 2011;18:649-54.

7 Boon AJ, Tans JT, Delwel EJ, et al. Dutch Normal-Pressure Hydrocephalus Study: randomized comparison of low- and medium-pressure shunts. J Neurosurg 1998;88:490-5.

8 Lemcke J, Meier U, Müller $C$, et al. Is it possible to minimize overdrainage complications with gravitational units in patients with idiopathic normal pressure hydrocephalus? Protocol of the randomized controlled SVASONA trial (ISRCTN51046698). Acta Neurochir Supp/ 2010;106:113-15.

9 Lemcke J, Meier U. Improved outcome in shunted iNPH with a combination of a Codman Hakim programmable valve and an Aesculap-Miethke Shunt Assistant. Cen Eur Neurosurg 2010;71:113-16.

10 Kiefer M, Meier U, Eymann R. Gravitational valves: relevant differences with different technical solutions to counteract hydrostatic pressure. Acta Neurochir Suppl 2006;96:343-7

11 Adams RD, Fisher CM, Hakim S, et al. Symptomatic occult hydrocephalus with "normal" cerebrospinal fluid pressure. A treatable syndrome. N Engl J Med 1965:273:117-26.

12 Evans WA. An encephalographic ratio for estimating ventricular enlargement and cerebral atrophy. Arch Neurol Psychiatry 1942;47:931-7.

13 Meier $U$, Bartels $P$. The importance of the intrathecal infusion test in the diagnosis of normal pressure hydrocephalus. J Clin Neurosci 2002;9:260-7.

14 Bech-Azeddine R, Gjerris F, Waldemar G, et al. Intraventricular or lumbar infusion test in adult communicating hydrocephalus? Practical consequences and clinical outcome of shunt operation. Acta Neurochir (Wien) 2005;147:1027-35.

15 Brean A, Eide PK. Assessment of idiopathic normal pressure patients in neurological practice: the role of lumbar infusion testing for referral of patients to neurosurgery. Eur J Neurol 2008;15:605-12.

16 Kahlon B, Sundbarg G, Rehncrona S. Lumbar infusion test in normal pressure hydrocephalus. Acta Neurol Scand 2005;111:379-84.

17 Kahlon B, Sundbarg G, Rehncrona S. Comparison between the lumbar infusion and CSF tap tests to predict outcome after shunt surgery in suspected normal pressure hydrocephalus. J Neurol Neurosurg Psychiatry 2002;73:721-6.

18 Tans JT. Differentiation of normal pressure hydrocephalus and cerebral atrophy by computed tomography and spinal infusion test. J Neurol 1979;222:109-18.

19 Damasceno BP, Carelli EF, Honorato DC, et al. The predictive value of cerebrospinal fluid tap-test in normal pressure hydrocephalus. Arq Neuropsiquiatr 1997:55:179-85. 
20 Marmarou A, Young HF, Aygok GA, et al. Diagnosis and management of idiopathic normal-pressure hydrocephalus: a prospective study in 151 patients. J Neurosurg 2005:102:987-97.

21 Sand T, Bovim G, Grimse R, et al. Idiopathic normal pressure hydrocephalus: the CSF tap-test may predict the clinical response to shunting. Acta Neurol Scand 1994;89:311-16

22 Wikkelso C, Andersson H, Blomstrand C, et al. Normal pressure hydrocephalus. Predictive value of the cerebrospinal fluid tap-test. Acta Neurol Scand 1986;73:566-73.

23 Aschoff A, Kremer P, Benesch C, et al. Overdrainage and shunt technology. A critical comparison of programmable, hydrostatic and variable-resistance valves and flow-reducing devices. Childs Nerv Syst 1995;11:193-202.

24 Aschoff A, Kremer P, Hashemi B, et al. The scientific history of hydrocephalus and its treatment. Neurosurg Rev 1999:22:67-93.

25 Klinge $P$, Marmarou A, Bergsneider $M$, et al. Outcome of shunting in idiopathic normal-pressure hydrocephalus and the value of outcome assessment in shunted patients. Neurosurgery 2005;57:S40-52.

26 Relkin N, Marmarou A, Klinge $P$, et al. Diagnosing idiopathic normal-pressure hydrocephalus. Neurosurgery 2005;57:S4-16.

27 Kiefer M, Eymann R, Komenda Y, et al. A grading system for chronic hydrocephalus. Zentralb/ Neurochir 2003:64:109-15.
28 Zemack G, Romner B. Seven years of clinical experience with the programmable Codman Hakim valve: a retrospective study of 583 patients. J Neurosurg 2000;92:941-8

29 Copeland KT, Checkoway H, McMichael AJ, et al. Bias due to misclassification in the estimation of relative risk. Am J Epidemiol 1977;105:488-95.

30 Benzel EC, Pelletier AL, Levy PG. Communicating hydrocephalus in adults: prediction of outcome after ventricular shunting procedures. Neurosurgery 1990;26:655-60.

31 Black PM. Idiopathic normal-pressure hydrocephalus. Results of shunting in 62 patients. J Neurosurg 1980;52:371-7.

32 Black PM, Ojemann RG, Tzouras A. CSF shunts for dementia, incontinence, and gait disturbance. Clin Neurosurg 1985;32:632-51.

33 Hellstrom P, Klinge P, Tans J, et al. The neuropsychology of iNPH: findings and evaluation of tests in the European multicentre study. Clin Neurol Neurosurg 2012;114:130-4.

34 Trigg R, Jones RW, Skevington SM. Can people with mild to moderate dementia provide reliable answers about their quality of life? Age Ageing 2007;36:663-9.

35 Seymour DG, Ball AE, Russell EM, et al. Problems in using health survey questionnaires in older patients with physical disabilities. The reliability and validity of the SF-36 and the effect of cognitive impairment. J Eval Clin Pract 2001:7:411-18 